\title{
Editorial
}

\section{Systolic Blood Pressure}

Intervention Trial and Statins,

a Story of Statistical Interaction

\author{
Wanzhu Tu Rajiv Agarwal \\ Indiana University School of Medicine, Indianapolis, IN, USA
}

The Systolic Blood Pressure Intervention Trial (SPRINT) showed that in older patients with higher risk of cardiovascular disease, intensive blood pressure (BP) control resulted in significantly improved cardiovascular outcomes and drastically reduced rates of mortality [1]. The SPRINT is a multicenter, open-label randomized controlled trial with 2 treatment arms: A standard treatment arm that aimed at reducing systolic BP to $<140 \mathrm{~mm} \mathrm{Hg}$, and an intervention arm that set the treatment goal to systolic $\mathrm{BP}<120 \mathrm{~mm} \mathrm{Hg}$. The primary outcome of the SPRINT study is the first incidence of myocardial infarction, acute coronary syndromes, stroke, heart failure, or death from cardiovascular causes. The study included more than 9,000 older patients with diverse levels of cardiovascular risks, excluding only those with a history of stroke or diabetes, and those with severely degraded kidney function (estimated glomerular filtration rate $<20 \mathrm{~mL} / \mathrm{min} / 1.73 \mathrm{~m}^{2}$ ). While the evidence is clear and convincing on the benefits of the intensive BP-lowering target in this broad population, the extent to which patients with specific comorbid conditions benefit from the intervention has not been fully explored.

\section{KARGER}

(c) 2019 S. Karger AG, Basel

E-Mail karger@karger.com

www.karger.com/ajn
An important condition that deserves special attention is chronic kidney disease (CKD). The association between hypertension and CKD is well documented, and it is thought that the 2 share mutually reinforcing pathophysiology that centers on the kidney's inability to dispose of sodium $[2,3]$. Adding to the hypertension-CKD conundrum is dyslipidemia. The benefit of statins to advanced CKD in cardiovascular protection was definitively established by the Study of Heart and Renal Protection (SHARP) trial [4]. The SHARP study was in large part responsible for the guidelines on dyslipidemia management in CKD by the Kidney Disease: Improving Global Outcomes 7 Lipid Work Group, leading to the recommendation of a statin/ ezetimibe combination for older adults with CKD (estimated glomerular filtration rate $<60 \mathrm{~mL} / \mathrm{min} / 1.73 \mathrm{~m}^{2}$ ), except for individuals with chronic dialysis or kidney transplantation. Despite these guidelines, a recent study showed that only about half the CKD patients who qualified for statin treatment were appropriately treated [5]. A logical question after the SPRINT study therefore is whether such a lipid-lowering therapy could be combined with the more aggressive BP goal set by SPRINT to achieve improved results. 
Fig. 1. The 2 diagrams illustrate a statistical interaction between SPRINT and statin use. Statin use modifies the effects of SPRINT on all-cause mortality (a) and cardiovascular mortality (b). The horizontal dotted line denotes HR of 1 . When the $95 \%$ CIs shown by the vertical bars cross the dotted line, the HR is not statistically significant. When CKD patients were not prescribed a statin, the $95 \%$ CIs of the adjusted HRs associated with the SPRINT intervention for both all-cause mortality and cardiovascular mortality covered the value 1 and thus were not statistically significant. In sharp contrast, both all-cause mortality and cardiovascular mortality were significantly lower when CKD patients were prescribed a statin. The $p$ values are associated with the significance tests for the interaction effects. HR, hazard ratio.

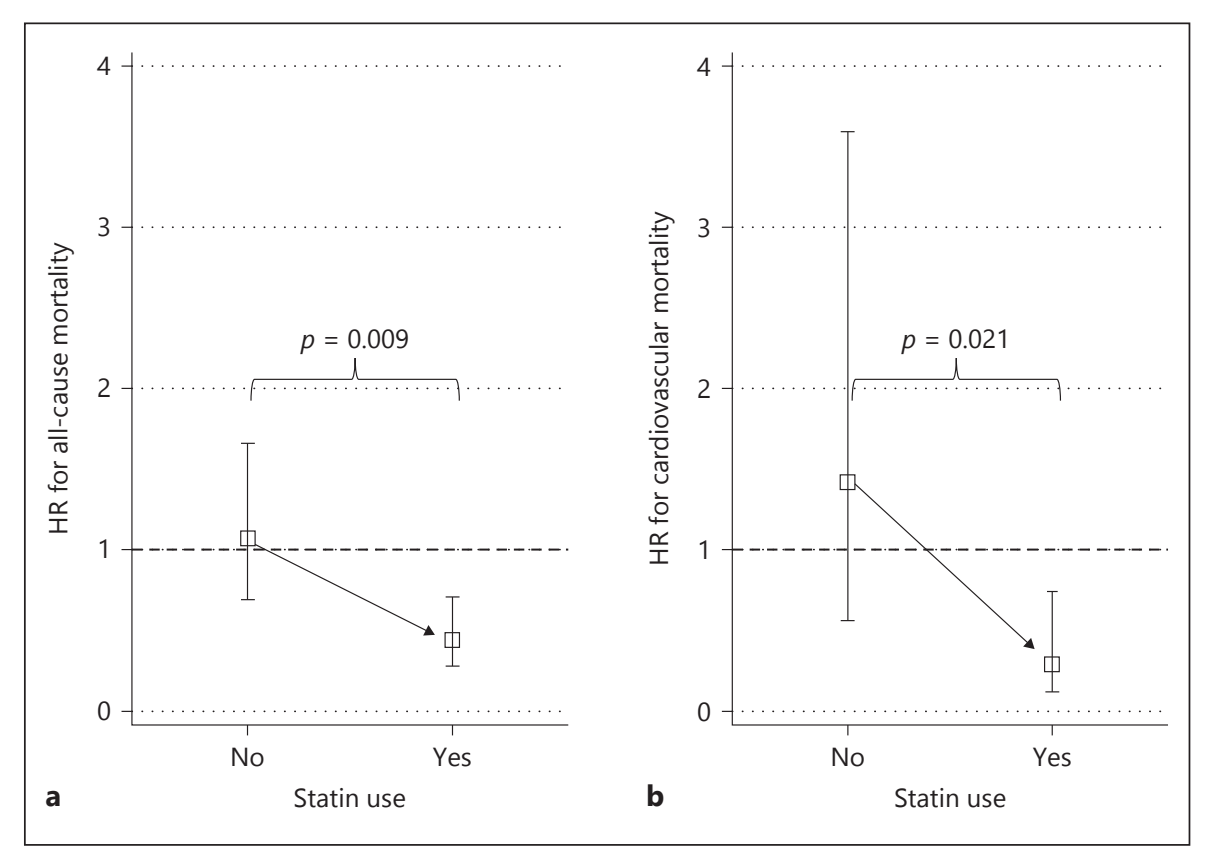

In the current issue of the American Journal of $\mathrm{Ne}$ phrology, Rivera et al. [6] attempted to address this question through a secondary analysis of the SPRINT data. Specifically, they identified 2,646 individuals from the SPRINT participants who had a diagnosis of CKD (stages 3 and 4). Importantly, only slightly over half of them (51\%, $n=1,354)$ were on statins. Rivera et al. [6] evaluated the modifying influences of statins on the relationship between the SPRINT intervention (setting a systolic BP target to $<120 \mathrm{~mm} \mathrm{Hg}$ ) and all-cause and cardiovascular mortality. Rivera et al. [6] reported that among those who had received statins, the adjusted hazard ratios (HRs) were 0.44 (95\% CI [0.28-0.71]) for all-cause mortality and 0.29 (95\% CI [0.12-0.74]) for cardiovascular mortality, while controlling for the influences of age, sex, race, cardiovascular disease history, smoking, aspirin, and baseline BP. The magnitudes of the reported effects are nothing short of remarkable as these hazard ratios suggest that adopting the lower systolic BP goal of 120 $\mathrm{mm} \mathrm{Hg}$, as opposed to the usual target of $140 \mathrm{~mm} \mathrm{Hg}$, corresponds to a reduction of all-cause mortality from 2.5 to 1.16 per 100 person-years, and a reduction of cardiovascular mortality from 0.92 to 0.28 per 100 personyears.

Interestingly, the authors did not observe a similar difference in the participants who had not been on statins at the baseline of the SPRINT study. In the non-statin group, the estimated adjusted HR associated with the lower BP goal was 1.07 (95\% CI [0.69-1.66]) for all-cause mortality, and 1.42 (95\% CI [0.56-3.59]) for cardiovascular-related mortality. The time-adjusted all-cause mortality rates were 2.01 per 100 person-years in the SPRINT group vs. 1.94 per 100 person-years in the control group. Rates of mortality due to cardiovascular causes were 0.52 per 100 person-years in the intervention group as opposed to 0.41 per 100 person-years in the control group.

The differential effects of the SPRINT intervention in statin and non-statin groups constitute a perfect example of statistical interaction, a situation where the causal effect of one intervention is influenced or modified by another factor. To contrast the drastically different mortality effects of the SPRINT intervention in patients who were on statins and those were not on statins, we highlighted Rivera's key findings graphically in Figure 1. While most clinical trials focus on testing the effects of the main interventions, secondary analyses provide excellent opportunities for assessing the important interaction effects.

The authors also compared the serious adverse events (SAEs) between the intervention and control patients in the statin and non-statin groups. Overall, they reported no statistically significant differences in SAEs between the 2 treatment arms within the statin and non-statin groups. The lack of statistical significance, however, could be a consequence of the much reduced sample size from the original SPRINT study, and thus should not be interpreted as evidence for lack of differences. The authors did not provide formal statistical comparisons on the SAEs 
between the statin and non-statin groups, although visual inspections suggested slightly more SAEs in the statin group (Table 3 of [6]). Polypharmacy and the potential for drug interactions make the elderly CKD population particularly vulnerable to the adverse effects of many classes of drugs including statins. Accordingly, prudence dictates that vigilance against the adverse effects of statins should be an ongoing concern for anyone with CKD treated with a statin.

As Rivera et al. [6] have correctly recognized, the post hoc nature of the analysis precludes the possibility of a causal interpretation for their main finding. To the authors' credit, they performed a secondary propensity score analysis, which generated results that are in support of the same conclusion. Similarly, statin use was determined in a nonrandomized fashion before the SPRINT randomization, that is, patients had either been on or not been on statins before they started their intensive or usual BP-lowering therapies of the SPRINT study. Data generated from such a post hoc analysis therefore do not lend itself to inference about the effects of adding statins to the aggressive BP-lowering therapy after it starts.
Despite these nuances, we applaud the effort of Dr. Rivera et al. [6] in assessing the merits of combining the SPRINT-intensive BP management goal with a lipidlowering therapy in patients with CKD. They presented perhaps the first evidence in support of such a strategy. Although their analysis is observational in nature, the large sample size and relatively consistent analytical results give credence to their findings. Is the evidence strong and compelling enough to mandate a sweeping change in the clinical guidelines? Perhaps not by the presently shown evidence alone. However, knowing that a large number of patients with CKD have benefited from a combined therapy of aggressive BP control and lipid management, with little evidence of harm, we would suggest that it is high time for a more rigorous assessment of the combined therapy. Definitive answers would come from a carefully designed clinical trial. Till such trials are done, when treating hypertension in those with CKD, the story of interaction between SPRINT and statin should at least trigger a thought of reducing cardiovascular burden not with $\mathrm{BP}$ reduction alone but also with statins.

\section{References}

1 Wright JT Jr, Williamson JD, Whelton PK, Snyder JK, Sink KM, Rocco MV, et al.; SPRINT Research Group. A Randomized Trial of Intensive versus Standard Blood-Pressure Control. N Engl J Med. 2015 Nov; 373(22):2103-16.

2 Bakris GL, Williams M, Dworkin L, Elliott WJ, Epstein M, Toto R, et al.; National Kidney Foundation Hypertension and Diabetes Executive Committees Working Group. Preserving renal function in adults with hypertension and diabetes: a consensus ap- proach. Am J Kidney Dis. 2000 Sep;36(3): 646-61.

3 Judd E, Calhoun DA. Management of hypertension in CKD: beyond the guidelines. Adv Chronic Kidney Dis. 2015 Mar;22(2):11622.

4 Baigent C, Landray MJ, Reith C, Emberson J, Wheeler DC, Tomson C, et al.; SHARP Investigators. The effects of lowering LDL cholesterol with simvastatin plus ezetimibe in patients with chronic kidney disease (Study of Heart and Renal Protection): a randomised placebo-controlled trial. Lancet. 2011 Jun; 377(9784):2181-92.

5 Mefford MT, Rosenson RS, Deng L, Tanner RM, Bittner V, Safford MM, et al. Trends in Statin Use Among US Adults With Chronic Kidney Disease, 1999-2014. J Am Heart Assoc. 2019 Jan;8(2):e010640.

6 Rivera M, Tamariz L, Suarez M, Contreras G. Modifying effect of statins on fatal outcomes in chronic kidney disease patients in the systolic blood pressure intervention trial: A post hoc analysis. Am J Nephrol. 2019;49:297-306. 